\title{
Patient comfort following thirty minutes of lens wear: piggy-back versus conventional rigid-lens wear
}

\section{WDH Gillan*}

The Anterior Eye Research Group, Department of Optometry, University of Johannesburg, PO Box 524, Auckland Park, 2006 South Africa

Received 24 October 2013; revised version accepted 17 March 2014

\begin{abstract}
Discomfort when wearing contact lenses, especially rigid contact lenses, is a common complaint amongst neophyte as well as experienced contact lens wearers. Wearing a piggy-back system of contact lenses has been shown to improve comfort and wearing time, especially in keratoconic subjects. Twenty two normal subjects wore a rigid lens or a piggyback system of lenses for thirty minutes and after
\end{abstract}

a thirty minute break swopped the mode of lens wear and wore the second modality for a thirty minute period. This study suggests that a piggyback lens system provides improved comfort compared to wearing a rigid lens on its own. The order of lens wear might, however, influence the perceived comfort. (S Afr Optom 2013 73(1) 3-7)

Key Words: Piggy-back contact lenses, silicon hydrogels, rigid contact lenses, contact lens comfort.

\section{Introduction}

Contact lens-related discomfort is a common reason for the discontinuation of lens wear ${ }^{1-3}$. Research suggests that up to $75 \%$ of contact lens wearers complain of discomfort and dryness while wearing their lenses, especially towards the end of the day ${ }^{4-7}$. Approximately $20 \%$ of lens wearers make use of ocular lubricants in an effort to alleviate their symptoms ${ }^{5,6}$. Fonn et al ${ }^{1}$ have shown subjects to have a significant preference for soft contact lenses over rigid lenses (primarily due to improved comfort when wearing a soft lens) while Cornish and Sulaiman ${ }^{8}$ state: "Initial discomfort has long been regarded as a major disadvantage of conventional, rigid gas permeable contact lenses, accounting for up to $30 \%$ of prospective wearers failing to adapt to RGP lenses...".

Bennett and Weissman ${ }^{9}$ state that Baldone ${ }^{10}$ initiated the use of piggy-backing a rigid lens over a soft contact lens. Other publications were produced advocating the same thing: wear a soft lens under a rigid lens to improve comfort ${ }^{11-14}$. Numerous publications have been produced reporting on the use of piggyback contact lens wear in keratoconus in an attempt to improve wearing time and patient comfort ${ }^{15-21}$. The primary reason for having to fit piggy-back systems to keratoconic patients was intolerance to wearing conventional rigid lenses. Improved comfort, wearing time and tolerance of lens wear in keratoconics were reported by several authors ${ }^{16-19,21}$. Little literature was found which investigates the effects of piggyback wear on comfort in normal subjects. Although it has been reported that comfort is improved when wearing a piggy-back system, just how much more comfortable is it?

The aim of this investigation was to evaluate levels of comfort after thirty minutes of conventional rigid 
lens versus piggy-back lens wear in normal subjects.

\section{Method}

Twenty two, non-contact lens wearing individuals, aged between 18 and 29 years (mode: 22 years of age), volunteered and gave written, informed consent to take part in this investigation. The tenets of the Declaration of Helsinki were adhered to throughout the study. Each subject underwent a preliminary evaluation to determine the parameters of the lenses that were to be fitted. Keratometry measurements were taken to provide an indication of the base curve that might need to be used for the first trial lens. For the rigid lens (RGP) fitting the initial lens was fitted $0.1 \mathrm{~mm}$ steeper than the flattest-K measurement. A flourescein and Burton lamp evaluation was then conducted to assess the adequacy of the lens to cornea relationship. The usual clinical requirements of an "on alignment" fit were adhered to. Relevant adjustments were made to the initial fit if deemed necessary. The piggy-back hydrogel (PB) lens used for each subject was a Biofinity silicone lens, base curve $8.6 \mathrm{~mm}$, with a low negative power. The hydrogel lens was assessed according to its centration and movement after a blink. The piggy-back modality consisted of the rigid lens worn during the rigid lens wearing session fitted "on top of" the Biofinity silicone hydrogel lens.

Each subject was expected to wear a lens modality (piggy-back or rigid lens) for thirty minutes during one wearing session, have a thirty minute break and then wear the opposite modality (rigid lens or piggy-back) for a further thirty minutes during a second wearing session. The subjects wore a lens modality on only one eye. The eye chosen to fit with a lens modality was the eye which had the least corneal astigmatism as determined by keratometry. All eyes fitted with a lens modality had $\leq-1.00 \mathrm{D}$ of corneal astigmatism except for one subject who had $-1.25 \mathrm{D}$. The order in which the subjects wore the different wearing modalities was randomized. At the end of each wearing session, subjects had to make a subjective decision regarding the comfort of the lens modality that had just been worn. A comfort scale ranging from 0 to 5 was used to assess the subject's opinion of lens comfort. Zero indicated severe discomfort, 1 moderate discomfort, 2 mild discomfort, 3 mild comfort, 4 moderate comfort and 5 complete comfort.

\section{Results}

Of the 22 subjects, 15 wore the RGP lens modality initially with seven subjects wearing the piggy-back modality initially. Table 1 shows the descriptive statistics for the different lens modalities. The Kolmogorov-Smirnov test for normal distribution suggests that the data for both groups were normally distributed ( $p=0.24$ and $p=0.37$ for the RGP and PG groups respectively).

Figure 1 shows box and whisker plots for the two groups. Each box indicates the $95 \%$ confidence interval on the relevant median. The blue line connects the relevant mean comfort level for each set of data (the improvement in mean comfort from RGP to PB wear is indicated). The relevant descriptive statistics relating to Figure 1 can be seen in Table 1 .

An independent samples $t$-test was conducted to investigate whether the means of the two groups were statistically different. It was shown that the means of the two groups were significantly different at the $95 \%$ level of confidence $(t=2.03, p=0.048)$.

Further analysis evaluated whether the order in which the two modalities were worn influenced the outcome. Table 2 shows results relating to the comfort level of the two modalities depending on which modality was worn first. Fifteen subjects wore the rigid lens first (RGP1) and the piggy-back system second (PB2) while seven subjects wore the piggyback system first (PB1) and the rigid lens second (RGP2).

Table 1. Descriptive statistics for the 22 subjects are provided for the different lens modalities. RGP and PB indicate the lens modality worn, SD is the standard deviation on the mean, 95\% CI indicates the $95 \%$ confidence interval for the mean and ND indicates whether the data were normally distributed or not (according to the Kolmogorov-Smirnov test) and "Yes" indicates that the data were normally distributed.

\begin{tabular}{|l|l|l|l|l|l|}
\hline Modality & Mean comfort & SD & $95 \%$ CI & Median comfort & ND \\
\hline RGP & 1.64 & 1.14 & 1.13 to 2.14 & 1.5 & Yes \\
PB & 2.32 & 1.09 & 2.0 to 3.0 & 2 & Yes \\
\hline
\end{tabular}




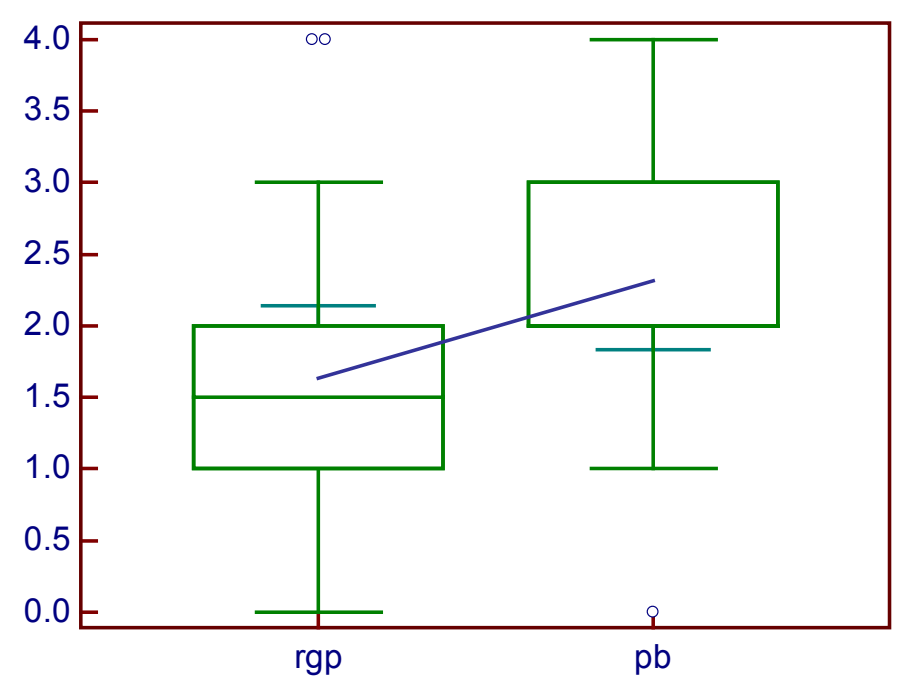

Figure 1. Box and whisker plots for the RGP and PB groups are shown. Each box indicates the $95 \%$ confidence interval on the medians with the whiskers extending from the lowest to highest scale (excluding far-out and extreme comfort scales). The blue line connects the relevant mean comfort level for each group. Circles indicate the extreme comfort levels (possible outliers) for each group.

Table 2. Comfort levels are shown for the order in which the two modalities were worn. RGP1 indicates the group of 15 subjects who wore the rigid lens first with the piggy-back system second (PB2) while PB1 indicates the seven subjects who wore the piggy-back system first and the rigid lens second (RGP2). The results of the $t$-test ( $t$ and $p$ ) between the RGP1 and PB2 subjects and the PB1 and RGP2 subjects are included. Significant differences are indicated.

\begin{tabular}{|l|l|l|l|l|l|l|}
\hline $\begin{array}{l}\text { Moda- } \\
\text { lity }\end{array}$ & $n$ & $\begin{array}{l}\text { Mean } \\
\text { comfort }\end{array}$ & SD & $t$ & $p$ & $\begin{array}{l}\text { Signi- } \\
\text { ficant }\end{array}$ \\
\hline RGP1 & 15 & 1.20 & 0.86 & 2.55 & 0.016 & Yes \\
PB2 & 15 & 2.13 & 1.13 & & & \\
PB1 & 7 & 2.71 & 0.95 & -2.55 & 0.803 & No \\
RGP2 & 7 & 2.57 & 1.13 & & & \\
\hline
\end{tabular}

The mean comfort levels for the rigid and piggyback system, and whether worn first or second, can be seen in Table 2. Table 2 also shows that the difference in mean comfort levels, when the rigid lens is worn first (RGP1 vs PB2), is significantly different while the difference in mean comfort levels when the piggy-back system is worn first (PB1 vs RGP2), is not significantly different. Further analysis comparing the mean comfort levels of the two lens systems worn first (RGP1 vs PB1) and the comfort levels of the two lens systems worn second (RGP2 vs PB2) shows that when the rigid lens is worn first (RGP1 vs PB1) there is a significant difference $(p=0.0014)$ in the comfort levels while when the rigid lens is worn second (PB2 vs RGP2), there is no significant difference $(p=0.41)$ in the mean comfort levels.

\section{Discussion}

Ocular discomfort is a common complaint of contact lens wearers, especially rigid lens wearers ${ }^{1-3}$. In an attempt to improve comfort and increase wearing time piggy-back lens systems can be used, with keratoconic patients often needing the improved comfort that a piggy-back system might provide ${ }^{16-19,21}$. This study was an attempt to elucidate whether piggy-back lens wear was, in fact, more comfortable than rigid lens wear and how much more comfortable the piggy-back system might be, in normal subjects.

Twenty two normal subjects wore a piggy-back system or a rigid lens for a thirty minute period followed by thirty minutes of wear of the alternative lens (a thirty minute rest period was provided between lens wear). The eye with the least amount of corneal astigmatism was the eye chosen to wear the lens. The order of lens wear (piggy-back first or rigid lens first) was determined randomly. Fifteen subjects wore the piggy-back system first with seven subjects wearing the rigid lens first.

Disregarding the order of lens wear, when all twenty two sets of data are compared the piggyback system was significantly more comfortable than rigid lens wear $(p=0.048)$. The comfort scale used in this study extended from 0 for severe discomfort to 5 for complete comfort. The mean comfort level for subjects wearing the piggy-back system was 2.32 with a mean comfort level for rigid lens wear of 1.64 (see Table 1). While a significant difference between the two wearing modalities exists the mean comfort level for the piggy-back system is between mild discomfort and mild comfort. No subjects reported complete comfort with either of the lens wear protocols. Figure 1 shows a visual representation of the data with the blue line between the two box and whisker plots connecting mean comfort levels for the 
two groups of subjects. When the order of lens wear (piggy-back first or rigid lens first) was considered, the results of this study suggest that the order of lens wear might play a role in the perceived comfort of the lens system being worn. The mean comfort level for fifteen subjects wearing the rigid lens first was 1.2 while the comfort level of the same fifteen subjects wearing the piggy-back system second was 2.13, a significant difference $(p=0.016)$. For the seven subjects who wore the piggy-back system first, the mean comfort level was 2.71 while the comfort level for those subjects wearing the rigid lens second was 2.57, which is not a significant difference (see Table 2 ). When comparing the comfort of subjects who wore the rigid lens first to those who wore the piggyback system first there is a significant difference ( $p$ $=0.0014)$ in comfort with the piggy-back system being more comfortable. When comparing subjects who wore the rigid lens second to those who wore the piggy-back system second there is no significant difference in mean comfort $(p=0.41)$. It might well be that the order of lens wear had an influence on the perceived comfort of the subjects and that this result needs to be taken into consideration when performing similar studies to this one.

Limitations of this study include: the number of subjects used was limited, only thirty minutes of lens wear for each lens system was conducted (it is possible that a longer wear period would influence these results as many subjects wearing the piggyback system started complaining of discomfort and dry eyes towards the end of the wearing period), the subjects should perhaps have worn the lens systems on a "cross-over" protocol which would allow for the possible influence that "order of wear" seems to have had in this study.

In conclusion, this study suggests that a piggy-back contact lens system is considered more comfortable than a rigid lens worn on its own. However, the order in which the two systems of lens wear are used and compared possibly plays a role in the perceived comfort of the particular lens system being worn.

\section{Acknowledgements}

My thanks to J Booysen, J Brand, N Kotze and C Lyle (final year optometry students) for the collection of the data used in this study.

\section{References}

1. Fonn D, Gauthier CA, Pritchard N. Patient preferences and comparative ocular responses to rigid and soft contact lenses. Optom Vis Sci 199572 857-863.

2. Riley C, Chalmers RL, Pence N. The impact of lens choice in the relief of contact lens related symptoms and ocular surface findings. Contact Lens Ant Eye 200528 13-19.

3. Chalmers RL, Begley CG, Moody K, Hickson-Curran SB. Contact lens dry eye questionnaire-8 (CLDEQ-8) and opinion of contact lens performance. Optom Vis Sci 2012 89 1435-1442.

4. Begley CG, Chalmers RL, Mitchell GL, Nichols KK, Caffery BA, Simpson T. Characterization of ocular surface symptoms from optometric practices in North America. Cornea 200120 610-618.

5. Begley CG, Caffery BA, Nichols KK, Chalmers RL. Responses of contact lens wearers to a dry eye survey. Optom Vis Sci 200077 40-46.

6. Chalmers RL, Begley CG. Dryness symptoms among an unselected clinical population with and without contact lens wear. Contact Lens Ant Eye 200629 25-30.

7. Doughty MJ, Fonn D, Richter D, Simpson T, Caffery BA, Gordan K. A patient questionnaire approach to estimating the prevalence of dry eye symptoms in patients presenting to optometric practices across Canada. Optom Vis Sci 1997 74 624-631.

8. Cornish R, Sulaiman S. Do thinner rigid gas permeable contact lenses provide superior initial comfort? Optom Vis Sci 199673 139-143.

9. Bennett ES, Weissman BA. Clinical contact lens practice. Philadelphia: Lippencott-Williams \& Wilkens, 2005

10. Baldone JA. The fitting of hard lenses onto soft contact lenses in certain diseased conditions. Contact Lens Med Bull 1973615.

11. Little L. Soft lenses in keratoconus. Optician 197116226.

12. Movani MR, Mody KK. The concept of the correction of high astigmatism with a combination of hard and soft lenses. Contacto 197620 31-33.

13. Westerhout D. "The combination lens" and therapeutic uses of soft lenses. Contact Lens 19734 3-22.

14. Gasson A, Morris J. The contact lens manual: a practical fitting guide, $2^{\text {nd }}$ ed. Oxford: Butterworth-Heinemann, 2000.

15. Smith KA, Carrell JD. High-DK piggyback contact lens over Intacs for keratoconus: a case report. Eye Contact Lens 200834 238-241.

16. O'Donnell C, Moldano-Codina C. A hyper-DK piggyback contact lens system for keratoconus. Eye Contact Lens 200430 44-48.

17. Özkurt Y, Otakan M, Gencaga T, Akkaya S. Contact lens visual rehabilitation in keratoconus and corneal keratoplasty. J Ophthalmol 2012 Article ID 832070 doi: 10.1155/2012/832070.

18. Acar BT, Vural ET, Acar S. Effects of contact lenses on the ocular surface in patients with keratoconus: piggyback versus clearkone hybrid lenses. Eye Contact Lens 201238 43-48.

19. Sengor T, Kurna SA, Aki S, Özkurt Y. High-DK piggyback 
contact lens system for contact lens-intolerant keratoconus patients. Clin Ophthalmol $20115331-335$.

20. Romero-Jiménez M, Santodomingo-Rubido J, FloresRodríguez P, González-Méijome JM. Which soft contact lens power is better for piggyback fitting in keratoconus? Contact Lens Ant Eye 201336 45-48.

21. Melia B, Youngs E, Stevenson G. Contemporary piggyback contact lens systems for keratoconus: case studies. Optom Prac 20078 11-18. 